\title{
Pandemia COVID-19: Artigo de Opinião na Perspetiva da Medicina Geral e Familiar
}

\section{Pandemic COVID-19: Opinion Article from the Perspective of General and Family Medicine}

Luís Miguel Rodrigues Nogueira ${ }^{1}$ Elisabete Borges²

\section{RESUMO}

A pandemia por COVID-19 afetou profundamente o nosso quotidiano e especialmente a saúde da população em geral.

O presente manuscrito é uma reflexão na perspetiva da Medicina Geral e Familiar sobre a prestação de cuidados médicos durante e após a pandemia por COVID-19.

Abordamos o que se alterou e os desafios que esperamos no futuro no que diz respeito à prestação de cuidados médicos.

PALAVRAS-CHAVE: COVID-19; Medicina Geral e Familiar; Pandemia

\section{ABSTRACT}

The COVID-19 pandemic has profoundly affected our daily lives and especially the health of the general population.

This manuscript is a reflection from the perspective of General and Family Medicine about provision of medical care during and after the COVID-19 pandemic.

We address what has changed and the challenges we expect in the future with regard to the provision of medical care.

KEYWORDS: COVID-19; Family Practice; Pandemics 


\section{INTRODUÇÃO}

E subitamente o mundo ficou em suspenso, a população ficou confinada em suas casas, redobramo-nos em medidas de higiene e desinfeção, encerram-se serviços, indústrias, escolas e creches... A pandemia por COVID-19 trouxe profundas mudanças no nosso quotidiano, na sociedade, economia e alvoroçou a saúde geral da população.

As instituições prestadoras de cuidados de saúde tiveram de se reorganizar de uma forma nunca antes vista e assistiu-se a um verdadeiro espírito de missão por parte de todos os profissionais de saúde envolvidos na luta contra esta infeção.

Este novo paradigma tem profundas consequências na saúde na população em geral, não só pelo surgimento de uma nova infeção, com tantos aspetos ainda desconhecidos, mas também, por todas as alterações ocorridas, funcionamento e dinâmica das instituições de saúde. Parece que de repente só se pensa na COVID-19 e deixamos de nos preocupar com todos os restantes problemas de saúde. Foram suspensas todas as consultas programadas de vigilância, e mesmo o número de consultas médicas de urgências diminuíram drasticamente devido ao medo da população em se dirigir às instituições de saúde.

\section{O QUE MUDOU E QUAIS OS DESAFIOS FUTUROS DA MGF?}

A especialidade de MGF assume-se como o primeiro contacto médico dos utentes com o sistema de saúde, gerindo todos os problemas de saúde independentemente das características pessoais da pessoa e de uma forma longitudinal e continuada no tempo. Os médicos de família são portanto, médicos pessoais com responsabilidades específicas na saúde da comunidade, promovendo a saúde, prevenindo a doença e providenciando cura, cuidados ou paliação. ${ }^{1}$ Ora, nesta época de pandemia por COVID-19 o acompanhamento dos utentes pelo seu MF, no que respeita a todos os outros "problemas não COVID-19", apesar de todos os esforços delineados, deixou de ser o ideal.

De repente fomos obrigados a privilegiar a atividade assistencial não presencial com recurso a consulta por telefone, videochamada, e-mail, ou seja, vimo-nos implicados numa "medicina digital", algo a que a MGF, especialidade médica que tem como base o estabelecimento de uma relação de proximidade com a população em geral, não está acostumada, e os utentes também não.
Sendo assim, talvez estejamos a correr o risco de um agravamento do estado de saúde da população em geral no que respeita, por exemplo, ao controlo de diversas doenças crónicas como são as doenças cardiovasculares (primeira causa de morte em Portugal), as doenças oncológicas, a doença renal, doença mental, etc. Também os grupos vulneráveis, como são as crianças e as grávidas, deixaram de ter um seguimento tão regular como até então, o que se pode traduzir em maiores riscos para a saúde por conta de diagnósticos mais tardios e menor aconselhamento.

De realçar ainda as doenças mentais, como são a depressão e ansiedade, que não só podem ter sido agravadas pelo isolamento social que assistimos hoje, como também pela menor acessibilidade/acompanhamento médico tradicional.

Relativamente aos rastreios oncológicos (rastreio do cancro colorretal, cancro do colo do útero, cancro de mama), estes ao serem suspensos temporariamente levarão a uma menor taxa de deteção e atraso na referenciação aos cuidados de saúde secundários, o que poderá originar um pior prognóstico.

Com o incremento inexorável da telemedicina será um desafio para os médicos fazer a monitorização do estado de saúde dos seus doentes crónicos, ${ }^{2}$ nomeadamente os idosos, polimedicados e com múltiplas comorbilidades que não estão acostumados a utilizar as plataformas eletrónicas de interação com os recursos de saúde disponíveis.

Como já referido, também as consultas de doença aguda diminuíram drasticamente porque os doentes têm medo de serem infetados e por isso recorrem menos aos serviços de saúde. Se anteriormente se assistia a uma sobrecarga de serviços de urgência, com problemas que não eram sequer urgentes, agora verifica-se exatamente o contrário. Não estarão os doentes a desvalorizar problemas graves e que devem ser orientados o quanto antes?

De notar ainda que o medo de contrair a infeção por COVID-19 não é exclusivo dos utentes, mas também nós médicos o sentimos. O medo poderá influenciar a nossa prestação como cuidadores quer pela pressão psicológica inerente a que estamos sujeitos, quer pela dificuldade em manter constante distanciamento físico de segurança com o doente a ser observado. O exame físico é uma parte importante da consulta de MGF pois não só contribui para informação diagnóstica, mas é um instrumento terapêutico por si só dado que contribui para melhor relação médico-doente. ${ }^{3}$ 
A pandemia tornou-nos talvez médicos mais distantes dos nossos doentes, além de grande parte das consultas serem à distância, usamos máscaras e fatos complexos em que só mostramos o olhar, não se vê o sorriso, a expressão facial. Ou seja, alterou-se a comunicação não-verbal, componente tão determinante na relação médico-doente. ${ }^{4}$

Com o retorno à "nova normalidade" precisamos de nos reinventar, de nos esforçar ainda mais para compensar o que possa ter ficado para trás nestes tempos difíceis. É necessário melhorar a acessibilidade, melhorar o seguimento de doentes crónicos, dos grupos vulneráveis e de risco, ter um especial cuidado nas ações de educação para a saúde, como são a promoção de estilos de vida saudáveis e a prevenção de doença e por fim, coordenarmo-nos eficazmente com as diferentes especialidades médico-cirúrgicas para em conjunto melhorar o seguimento dos nossos doentes. Talvez esta seja também uma boa oportunidade para termos um papel ainda mais ativo na promoção de uma maior literacia em saúde dos nossos doentes, esclarecendo por exemplo, sobre o que são problemas urgentes e que necessitam de cuidados médicos com maior brevidade, daqueles que não o são.

Assim, com este retomar das rotinas habituais esperam-nos tempos trabalhosos. Teremos de aumentar a nossa resiliência e capacidade de superar desafios para voltarmos novamente a ser "próximos" dos nossos utentes ao mesmo tempo que lidamos com a realidade da COVID-19 que talvez tenha vindo para ficar.

\section{RESPONSABILIDADES ÉTICAS}

CONFLITOS DE INTERESSE: Os autores declaram não possuir conflitos de interesse.

SUPORTE FINANCEIRO: O presente trabalho não foi suportado por nenhum subsídio ou bolsa.

PROVENIÊNCIA E REVISÃO POR PARES: Não comissionado; revisão externa por pares.

\section{ETHICAL DISCLOSURES}

CONFLICTS OF INTEREST: The authors have no conflicts of interest to declare.

FINANCING SUPPORT: This work has not received any contribution, grant or scholarship.

PROVENANCE AND PEER REVIEW: Not commissioned; externally peer reviewed.

\section{REFERÊNCIAS}

1. WONCA EUROPE. The European Definition of General Practice / Family Medicine. Ljubljana: WONCA; 2011. [accessed May 2020] Available from: https://www.woncaeurope.org/ page/definition-of-general-practice-family-medicine.

2. The Medical Futurist. Looking Back At COVID-19 From 2030 [accessed May 2020] Available from: https://medicalfuturist. com/looking-back-at-covid-19-from-2030/?utm_source=The\%20Medical\%20Futurist\%20Newsletter\&utm_campaign=4f9f158f33-EMAIL_CAMPAIGN_2020_05_19_Newsletter\&utm_medium $=$ email\&utm_term $=0$ _efd $6 a 3 \mathrm{~cd}$ 08-4f9f158f33-420632721.

3. Kelly MA, Freeman LK, Dornan T. Family Physicians' Experiences of Physical Examination. Ann Fam Med. 2019;17:304-10. doi: 10.1370/afm.2420.

4. Silva PR. A comunicação na prática médica: seu papel como componente terapêutico. Rev Port Clin Geral. 2008;24:50512. 